\title{
Psychosocial Factors and Diabetes
}

\author{
Authors \\ Bernhard Kulzer ${ }^{1}$, Christian Albus ${ }^{2}$, Stephan Herpertz ${ }^{3}$, Johannes Kruse ${ }^{4}$, Karin Lange ${ }^{5}$, Florian Lederbogen ${ }^{6}$, \\ Frank Petrak ${ }^{3}$
}

\begin{abstract}
Affiliations
1 Diabetes Center Bad Mergentheim, Research Institute of the Diabetes Academy (Forschungsinstitut der Diabetes Akademie - FIDAM), Mergentheim, Germany

2 Department of Psychosomatic Medicine and Psychotherapy, University of Cologne, Germany

3 Department of Psychosomatic Medicine and Psychotherapy, LWL-University Hospital of the Ruhr-University Bochum Germany

4 Department of Psychosomatics and Psychotherapy of the Justus-Liebig-University Gießen and Philipps University Marburg, Germany

5 Department of Medical Psychology, Hannover Medical School, Germany

6 Joint practice Weimer/Tabakhtory-Fard; Heidelberg
\end{abstract}

published online $\quad 12.08 .2021$
Bibliography

Exp Clin Endocrinol Diabetes 2021; 129: S91-S105

DOI 10.1055/a-1284-6524

ISSN $\quad 0947-7349$

(C) 2021. Thieme. All rights reserved.

Georg Thieme Verlag KG, Rüdigerstraße 14,

70469 Stuttgart, Germany

German Diabetes Association: Clinical Practice Guidelines

This is a translation of the DDG clinical practice guideline published in Diabetologie

2020; 15 (Suppl 1): S232-S248. DOI 10.1055/a-1194-2962

\section{Correspondence}

Prof. Dr. phil. Dipl. psych. Bernhard Kulzer

Diabetes-Zentrum Mergentheim, Forschungsinstitut

Diabetes-Akademie Bad Mergentheim (FIDAM GmbH)

Theodor-Klotzbücher-Straße 12

97980 Bad Mergentheim

Germany

kulzer@diabetes-zentrum.de

\section{Introduction}

For the therapy and long-term prognosis of people with diabetes mellitus, somatic and psychosocial factors play an equally important role. In diabetes therapy, the patient plays a decisive role, as he or she must implement the essential therapeutic measures of diabetes in his or her personal everyday life in a lasting and self-responsible manner. The prognosis of diabetes patients therefore depends to a large extent on the extent to which they succeed in doing so against the background of their social, cultural, family and professional environment. The following psychosocial factors are of central importance:

- Acquisition of knowledge and skills for self-treatment and their implementation in everyday life,

- Emotional and cognitive acceptance of diabetes,

- Coping with diabetes and its possible consequences in all affected areas of life and different stages of the disease (e. g. diabetic stress, acute and subsequent complications),

- Identification and modification of behavioral patterns that prevent successful self-treatment,

- Successful handling of crises and/or problems related to the illness (e. g. psychological problems such as depression, anxiety, eating disorders).
The present recommendations for psychosocial interventions in diabetes mellitus refer only to adult patients.

The important areas of "social legal consequences of diabetes" (e.g. profession, driver's license, Disabilities Act) or "diabetes and migrants" are not covered in this guideline.

\section{Psychoeducation/Diabetes Selfmanagement Education}

\section{Definition}

- Structured diabetes selfmanagement education is a systematic and goal-oriented process in which a person is enabled, through the acquisition of knowledge and skills about the disease and its treatment, to integrate diabetes into his or her own life in the best possible way on the basis of his or her own decisions, to avoid acute or long-term negative consequences of diabetes and to maintain quality of life. It represents an essential and indispensable element of diabetes therapy.

- Structured education and treatment programs for people with diabetes are characterized by a comprehensible, goal-oriented 
structure in the teaching of the training content. As a rule, this means that the essential contents and goals as well as the methodology and didactics are described in a curriculum and corresponding documents, such as working materials, and are available for both the educators and persons being educated. Diabetes education should be integrated into the treatment of diabetes; this is also expressed by the term "structured education and treatment programs".

\section{Target group}

- The target group for diabetes education courses are all people affected by the metabolic disease diabetes mellitus and their relatives or caregivers.

- For patients who cannot implement their diabetes therapy independently and safely (e.g. people with cognitive disabilities, geriatric patients), structured education should also be offered to the appropriate caregivers (e. g. care staff).

- Diabetes education should be offered immediately after diagnosis and as the disease progresses.

\section{Goals of structured diabetes education and treatment programs}

- The following topics are among the central elements of contemporary diabetes education concepts: information about diabetes, possible comorbidities, complications and suitable therapy measures. Furthermore, it is vital to practice skills for implementing the therapy and self-treatment in everyday life, to motivate patients to adopt a health-promoting lifestyle and their social competence, and to promote coping skills and strategies for maintaining quality of life. Since many patients have difficulties implementing the therapy measures, it is important to ensure that sufficient space is provided in the diabetes education for support with diabetes-specific problems. Adequate support for these problems should be developed together with the patient (see info box "Goals of diabetes training").

- In order to achieve these goals, the selection of diabetes education courses must take into account the type of diabetes, the form of therapy, the level of knowledge and education to date, the risk profile and prognosis of the disease, the motivational, cognitive, behavioral, psychological and special cultural prerequisites of the patients, as well as special problem situations related to the disease.

\section{GOALS OF DIABETES EDUCATION}

- Information and education about the disease diabetes, possible concomitant diseases and complications

- Support in accepting the disease, development of motivation for treatment and support for personal responsibility in handling diabetes

- Promoting the patient's active, self-determined role in the therapy process, supporting the patient's independent decision-making ability
- Promoting therapy-supporting measures relevant to everyday life (e.g. nutrition, exercise)

- Support in the formulation of treatment goals

- Imparting knowledge, skills and abilities for active implementation of suitable therapy measures for the treatment of diabetes, possible comorbidities diseases and complications

- Avoidance of acute and late complications of diabetes

- Promotion of social competence, coping skills and strategies to maintain quality of life

- Review of knowledge, skills and abilities related to the patient's self-treatment behavior

- Assistance in obtaining social support in connection with the disease (e. g. family members, self-help groups)

- Practice-relevant support for problems in connection with the implementation of diabetes therapy

- Avoidance of negative social consequences, discrimination due to the disease

\section{Forms of Diabetes Education}

- Basic education: Basic education and treatment programs, which should be conducted as soon as possible after the manifestation of diabetes or the switch to a different therapy regime, are designed to work with the patient to develop basic knowledge and skills for implementing diabetes therapy, making informed decisions, and managing the disease. The training should take place in a structured form in homogeneous groups and include the contents described in the appendix.

- Repeat, refresher and supplementary education: A single education session is unlikely to have a lifelong effect on the patient's diabetes self-management, and the effect of education diminishes with increasing duration of the follow-up period. So-called repetition, refresher, or supplementary training measures have the primary goal of supporting patients with difficulties in implementing therapy in everyday life and offering concrete assistance with problems related to diabetes (e.g., lack of knowledge, skills, complications, problems in everyday life), taking into account changes in the course of life.

- Problem-specific education and treatment programs: These are aimed at patients in special, diabetes-specific problem situations (e.g. the occurrence of complications or special problems such as hypoglycemia problems). In contrast to basic education programs, these group programs target specific patient groups.

- Individual training: in certain situations, individual training can also be useful. 


\section{INDICATIONS FOR DIABETES EDUCATION}

Indications for basic diabetes education:

Every person with diabetes mellitus should receive basic diabetes education as part of diabetes therapy. This should be offered immediately after diagnosis and, if necessary, as the disease progresses.

The indication for a follow-up or repeat education may be given if

- Significant changes in therapy become necessary (e.g. change to insulin therapy)

- The patient has significant problems with the implementation of diabetes therapy in everyday life (e. g. change of lifestyle, insulin dosage, management of hypoglycemia, nutrition, insulin resistance due to weight gain)

- The set therapy goals are not achieved (e. g. permanentlyelevated blood glucose, blood pressure or blood fat values, hypoglycemia, body weight/BMI, waist circumference)

- There is a permanent deterioration of the metabolic situation (e. g. measured by the HbA1c value, recurrent hypoglycemia)

- Special knowledge and skills are required for special life situations (e. g. job, illness, travel)

- Complications of diabetes occur that require special knowledge and skills of the patient (e.g. neuropathy, sexual disorders, diabetic foot, nephropathy, retinopathy, cardiovascular events)

- Significant motivation problems occur during the implementation of diabetes therapy

- Special living conditions exist which make the implementation of the therapy significantly more difficult (e. g. physical or psychological disabilities, migration)

The indication for problem-specific education and a treatment program can be given if

- The patient has to implement a specific, new form of therapy in everyday life (e. g. insulin pump therapy, continuous glucose monitoring)

- Significant problems occur because of acute complications (e. g. hypoglycemia perception disorder)

- Significant problems occur because of complications (e. g. neuropathy, sexual disorders, diabetic foot, nephropathy, retinopathy, cardiovascular events)

- Special situations occur in everyday life that make it difficult to enforce the therapy (e. g. shift work, fasting, extensive physical exercise)

- Special social (e. g. job) or psychological problems (e. g. depression) associated with diabetes
The indication for an individual education may be given if

- Group education courses are not available

- A patient is unsuitable for group education (e. g. language problems)

- Specific contents of diabetes therapy must be taught straight (e.g. in case of manifestation of type 1 diabetes, change to insulin therapy for type 2 diabetes)

- Individual problems occur in connection with diabetes (e. g. adherence problems, special therapy recommendations)

- Special, significant problems occur in connection with the diabetes therapy (e.g. difficulties with the insulin regimen, insulin pen)

- There are special situations in everyday life that make it difficult to implement the therapy

\section{Certified education and treatment programs}

A list of education programs for adults with diabetes mellitus certified by the Federal Office for Social Security (Bundesamt für Soziale Sicherung, BAS) and the German Diabetes Society (Deutsche Diabetes Gesellschaft - DDG) is provided below ( $>$ Tab. 1).

\section{Cognitive Impairments and Dementia}

Cognitive impairments are presented as group F0 in the International Classification of Mental Disorders (ICD-10) of the World Health Organization (WHO). This group includes mental illnesses with a verifiable etiology in a cerebral disease, brain injury or other damage leading to brain dysfunction. The dysfunction or disorder can be primary such as illnesses, injuries or dysfunctions that directly or specifically affect the brain, or secondary as in systemic diseases or disorders that affect the brain as one of many other organs or body systems.

In patients with diabetes, transient cognitive impairments due to acute hypo- or hyperglycemia must be distinguished from persistent mild, everyday functions that do not restrict cognitive performance and clinically-relevant dementia.

\section{Transient cognitive decline in performance}

Patients with diabetes should therefore be informed about both acute cognitive impairment due to hypoglycemia and pronounced hyperglycemia (blood glucose levels above $250 \mathrm{mg} / \mathrm{dl}$ ). Possible consequences for school, study and work performance as well as safety risks at work and in road traffic need to be discussed:

- Cognitive performance is impaired in patients with diabetes in all age groups with blood glucose levels below approximately $60 \mathrm{mg} / \mathrm{dl}$. 
- Tab. 1 Education programs certified in Germany by the Federal Office for Social Security (Bundesamt für Soziale Sicherung, BAS) or the German Diabetes Society (Deutschen Diabetes Gesellschaft - DDG).

\begin{tabular}{|c|c|c|c|}
\hline \multirow{2}{*}{ Title of the education program Authors and source } & \multirow{2}{*}{ Evidence class } & \multicolumn{2}{|c|}{ Approval } \\
\hline & & BAS & DDG \\
\hline Treatment and education program for intensified insulin therapy (for type 1 diabetes) & Ila & yes & yes \\
\hline $\begin{array}{l}\text { PRIMAS: Living with type } 1 \text { diabetes - A education and treatment program for an autonomous life with type } 1 \\
\text { diabetes }\end{array}$ & $\mathrm{Ib}$ & yes & - \\
\hline Training program for people with typ2 diabetes who do not inject insulin & Ila & yes & Yes \\
\hline $\begin{array}{l}\text { More diabetes self-management for Type 2: A education and treatment program for people with non-insulin- } \\
\text { dependent type } 2 \text { diabetes MEDIAS } 2 \text { BASIC) }\end{array}$ & $\mathrm{lb}$ & yes & No \\
\hline $\begin{array}{l}\text { Treatment and education program for patients with type } 2 \text { diabetes who inject insulin (conventional insulin } \\
\text { therapy) }\end{array}$ & Ila & yes & - \\
\hline $\begin{array}{l}\text { Treatment and education program for people with typ } 2 \text { diabetes who inject normal insulin (preprandial } \\
\text { insulin therapy) }\end{array}$ & Ila & yes & No \\
\hline $\begin{array}{l}\text { More diabetes self-management type 2: Education and treatment program for people with type } 2 \text { diabetes } \\
\text { and conventional intensified insulin therapy (MEDIAS } 2 \text { ICT) }\end{array}$ & $\mathrm{lb}$ & yes & yes \\
\hline Type 2 diabetes under discussion - education program for people with who do not inject insulin & III & yes & No \\
\hline Diabetes \& behavior - education program for people with type 2 diabetes who inject insulin & $\mathrm{Ilb}$ & yes & No \\
\hline LINDA - education program for people with type 1 or type 2 diabetes & Ila & yes & No \\
\hline $\begin{array}{l}\text { For feet's sake (BARFUSS) - Structured treatment and education program for people with diabetes and diabetic } \\
\text { foot syndrome }\end{array}$ & IIb & nein & yes \\
\hline Blood glucose perception training (BGAT III) & $\mathrm{Ib}$ & yes & yes \\
\hline $\begin{array}{l}\text { HyPOS - how to better detect, prevent and manage hypoglycemia: A structured education and treatment } \\
\text { program for insulin-dependent diabetes patients with hypoglycemic problems }\end{array}$ & $\mathrm{Ib}$ & yes & yes \\
\hline $\begin{array}{l}\text { Stay fit and grow older with diabetes. Structured education program for type } 2 \text { diabetics of advanced age who } \\
\text { inject insulin }\end{array}$ & Ib & yes & yes \\
\hline DiSko-Education (DiSko: how to join people with diabetes with sports) & Ila & yes & yes \\
\hline FLASH education and treatment program for people who use flash glucose monitoring & $\mathrm{Ib}$ & nein & yes \\
\hline
\end{tabular}

- The impairments affect processing speed, complex psychomotor tasks, and emotional status, each of which is closely related to glucose levels.

- Acutely-elevated blood glucose levels can also impair concentration and cognitive performance. This has a negative impact on working memory, attention and emotional mood.

\section{Cognitive impairment and mild cognitive impairments $(\mathrm{MCl})$}

It is not yet possible to make reliable statements on the prevalence of $\mathrm{MCl}$ in diabetes on the basis of the available data due to the heterogeneity of the methods, operationalization of cognitive performance and the patient populations involved.

\section{Dementia in diabetes}

Patients with type 2 diabetes should be informed about the association between a long-term inadequate metabolic control and an increased risk of dementia.

- Patients with type 2 diabetes have a 2 to 4 -fold increased risk of vascular dementia and a 1.5 to 2 -fold increased risk of Alzheimer's dementia.

\section{Risk factors for cognitive impairment in diabetes}

- Chronic hyperglycemia

- Diabetes duration

- Age
- Hypertension

- Lipid metabolic disorders

- Obesity

- Stroke

- Depression

- Smoking

- Inflammatory processes

- Rheological factors

- Dysregulation of the hypothalamic-pituitary-adrenal (HPA) axis

- Hypoglycemia

\section{Screening and Diagnostics}

The early diagnosis of cognitive impairment is relevant in diabetes to ensure that a patient is still able to responsibly and reliably coordinate his or her (insulin) therapy and the appropriate diet on his or her own. In the risk group of elderly people (older than 65 years and with prolonged diabetes) and with cardiovascular comorbidity and clinical evidence of $\mathrm{MCl}$, annual dementia screening should be performed.

- Possible indications of cognitive impairment:

- Hypoglycemia due to incorrect insulin dosage,

- Considerable fluctuations in the glucose level due to incomplete medication and/or malnutrition,

- Difficulties in measuring blood glucose, handling the insulin pen, calculating insulin dose, or responding appropriately to hypoglycemic symptoms. 
- The diagnosis of dementia is an extremely serious information for affected persons and their relatives, which can lead to great psychological stress. Before the diagnosis is made, the highest possible diagnostic certainty should therefore be ensured. In addition to information about the diagnosis, the patient, relatives and the environment should also be given comprehensive information on support and counseling services.

- Indications are provided by patients' self-reported memory problems, e.g., difficulty finding words, retaining things, retrieving things, or concentrating.

- General, established short tests for the diagnosis of dementia can be used as screening instruments or as orientation aids in monitoring the course of the disease and for assessing its severity:

- Dementia detection test (DemTect)

- Mini mental status test

- Clock sign test

- Test for early detection of dementia separate from depression

- Reisberg scales for external assessment

- Furthermore, the recording of everyday activities with the Barthel Index or the Instrumental Activities of Daily Living (IADL) scale is useful to assess the need for help in general and in diabetes therapy.

- Further in-depth neuropsychological early and differential diagnosis should be guided by the recommendations of the evidence-based guidelines on dementia.

\section{Therapy of diabetes with cognitive impairments}

- When choosing therapy goals and principles, the heterogeneity of the physical and mental performance and the associated risks of this patient group should be taken into account.

- In elderly persons with significant cognitive and/or functional impairment, HbA1c levels at which hypoglycemia is safely avoided should be targeted. However, hyperglycemia leading to the exacerbation of geriatric syndromes or dehydration should also be avoided, i.e., HbA1c values around 8\% (64 mmol/ mol) should be aimed for. Simple therapy concepts and dietary recommendations adapted to daily routines should protect against excessive demands and maintain quality of life.

- Simple therapy concepts and nutritional recommendations adapted to daily routines should protect against feeling overwhelmed and should maintain the quality of life. Simple antihyperglycemic therapies carried out by caretakers with little stress on the patients make more sense here than concepts that are too demanding for both parties.

- In dementia patients, HbA1c values are secondary, but syndromes and hypoglycemic events that impair the quality of life must be avoided.

\section{Addictions - Alcohol and Tobacco}

\section{Alcohol}

Alcohol addiction is no more common in people with diabetes than in the general population. People with a dependency disorder often have comorbid mental illnesses.

\section{Interaction of alcohol consumption and diabetes}

- Moderate alcohol consumption has a protective effect on the development of type 2 diabetes compared to low consumption or abstinence.

- The level of insulin resistance is lowest in people with regular moderate alcohol consumption but increases in both heavy drinkers and people who abstain from alcohol.

- People with diabetes should be educated that moderate, low-risk alcohol consumption is compatible with good metabolic control and diabetes prognosis, but that increased alcohol consumption increases the risk of hypoglycemia. About one in five severe hypoglycemic events that lead to hospitalization are caused by alcohol consumption.

- People with diabetes with a risky alcohol consumption or an alcohol dependency should also be educated about the dangers of alcohol, especially with regard to poorer metabolic control and the risk of co-morbid diseases.

\section{Screening and diagnostics of alcohol addiction/ abuse or harmful use}

Because of the negative effects of substance addiction on diabetes therapy, early diagnosis is important. Part of the clinical picture is that affected persons deny an alcohol dependency or alcohol abuse and its negative effects for a long time.

\section{Screening}

People with diabetes should be asked regularly - at least once a year - about their alcohol consumption, and if there is any suspicion of risky or harmful alcohol consumption, screening or further diagnosis of harmful alcohol consumption or alcohol dependency should be carried out.

- In practice, the four questions of the CAGE-G questionnaire (CAGE-G) have proven to be useful for screening harmful alcohol consumption (see info box "CAGE-G questions...")

- Munich alcoholism test (MALT)

- Trier alcoholism inventory (TAI)

- Lübeck Alcohol Addiction and Abuse Screening Test (LAST)

The Alcohol Use Disorders Identification Test (AUDIT, German versions: AUDIT-G-L and AUDIT-G-M or the Brief Alcohol Screening Instrument for primary Care [BASIC]) aim to detect risky or harmful alcohol consumption.

\section{Diagnostics}

- The questions for a diagnostic clarification according to the ICD-10 criteria can be found in the info box "Questions on alcohol addiction".

- Laboratory indicators of alcohol addiction such as gammaglutamyl transferase (gamma-GT), carbohydrate-deficient transferrin (CDT) or mean corpuscular volume of red blood cells (MCV) can support a diagnosis, but are not sensitive enough on their own to detect patients with alcohol addiction in clinical practice.

- Furthermore, it may be useful to additionally screen for somatoform disorders, depression or anxiety disorders in those affected, since many people with a dependency problem have comorbid mental disorders. 


\section{CAGE- G QUESTIONS}

For screening of harmful alcohol consumption

1 Have you ever felt you should cut down on your drinking?

2 Have people annoyed you by criticizing your drinking?

3 Have you ever felt bad or guilty about your drinking?

4 Have you ever had a drink first thing in the morning to steady your nerves or get rid of a hangover?

Evaluation: If at least one of the questions is answered with "Yes", there is a suspicion of an alcohol problem; if two or more "Yes" answers are given, harmful consumption or alcohol addiction is likely.

\section{QUESTIONS ON ALCOHOL ADDICTION}

For a diagnostic clarification according to the ICD-10 criteria

1. Do you (often) feel a strong urge, a kind of uncontrollable desire to drink alcohol?

2. Does it happen that you can't stop drinking once you start?

3. Do you sometimes drink in the morning to relieve an existing nausea or tremor (e.g. of your hands)?

4. Do you increasingly need more alcohol before you achieve a certain (desired) effect?

5. Do you change your daily plans to be able to drink alcohol or do you set up your day so that you can consume alcohol regularly?

6. Do you drink even though you feel that alcohol consumption is harming you physically, psychologically, or socially?

Evaluation: An alcohol addiction is present if at least three of these criteria occurred repeatedly for one month or within twelve months. In addition to a physical examination, an individual diagnosis requires a detailed anamnesis of the drinking habits and accompanying physical and psychological problems.

\section{Therapy of alcohol-related diseases}

Because of the increased health risks associated with addiction and its negative impact on diabetes treatment, treating addiction is of particular importance for patients with diabetes. For forms of low to moderate alcohol consumption or occasional "binge drinking", short-term interventions (behavioral medical interventions) are appropriate, while for more severe forms of alcohol addiction/ abuse, special addiction therapies are needed. Every person with diabetes with an alcohol-related addiction disorder should be offered the possibility to participate in an appropriate therapeutic intervention to treat the addiction disorder.

\section{Smoking}

In Germany, about $21 \%$ of people with type 1 diabetes and $13 \%$ with type 2 diabetes smoke. The proportion of smokers has declined in recent years.

\section{Interaction of smoking and diabetes}

- People with an increased risk of type 2 diabetes should be advised that smoking increases the risk of developing impaired glucose tolerance as well as the manifestation of type 2 diabetes.
- People with diabetes should be educated about the fact that smoking, as an independent risk factor, increases the risk of cardiovascular diseases such as stroke, heart attack or coronary heart disease.

- Smoking is an independent risk factor for increased mortality for people with diabetes.

- People with diabetes who smoke should be educated about the positive health effects of not smoking.

\section{Screening}

- Because of the importance smoking plays in the prognosis of diabetes, it is important that every person with diabetes is asked regularly - at least once a year - about their tobacco consumption. It must be taken into account that those affected often deny their addiction and underestimate the negative effects in terms of the risk of diabetes-related or associated secondary and concomitant diseases.

- The degree of addiction depends, among other things, on the number of cigarettes smoked daily (cigars, pipe), at what time the morning cigarette is smoked and the daily smoking profile, the depth of inhalation and the brand of cigarette used.

- The Fagerström test (FTND-G) (see info box “Fagerström test") is recommended for determining nicotine addiction. This test allows the severity of the addiction to be assessed on the basis of 6 questions, which can be asked orally or in writing in the form of a short questionnaire.

\section{Diagnostics}

- A detailed history of smoking habits (number of cigarettes smoked daily, daily smoking profile) is important for the diagnosis of a tobacco addiction. For a diagnostic clarification according to the ICD-10 criteria, the questions listed in the "Nicotine addiction" info box are suitable in practice.

- Patients with a tobacco addiction should also be screened for somatoform disorders, depression and anxiety disorders, since many people with an addiction problem have comorbid mental disorders.

\section{FAGERSTRÖM TEST}

\section{Questions about nicotine addiction}

1. How soon after you wake up do you have your first cigarette?

2. Do you find it difficult to to refrain from smoking in places where smoking is prohibited (e.g. in church, at the library, at the cinema, etc.)?

3. Which cigarette would you hate most to give up?

4. How many cigarettes do you smoke on average per day?

5. Do you smoke more often during the first hours after waking than during the rest of the day?

Evaluation: For the FTND-G a sum value is determined by adding the values for all six items. Higher values indicate a higher degree of tobacco addiction. In addition, cut-off values are also used to differentiate smokers with low, medium and severe tobacco addiction. Three categories are formed for this purpose: 1) 0-2 points: no or very low tobacco addiction, 2) 3-4 points: low addiction, and 3) 5-10 points: medium to high addiction. 


\section{NICOTINE ADDICTION}

Diagnostic questions on nicotine addiction according to the ICD-10

1. Do you (often) feel a strong urge, a kind of uncontrollable desire to smoke?

2. Do you have limited control over when to start and stop smoking and how much you smoke?

3. Do you have withdrawal symptoms such as irritability, nervousness, trembling, increased appetite when you want to limit or quit smoking?

4. Do you have to smoke more and more or in shorter intervals in order to achieve a constant effect of smoking or to prevent the above-mentioned withdrawal symptoms of smoking?

5. Do you change daily plans to be able to smoke or do you set up your day so that you can smoke regularly?

6. Do you smoke even though you feel that it is damaging to you physically, psychologically or socially?

Evaluation: A tobacco addiction exists if at least three of these criteria occurred repeatedly for one month or within twelve months.

\section{CONSULTATION PROCEDURE}

Counselling steps for abstaining from nicotine: Principles for counselling nicotine addiction:

- Asking about the current smoking status ("Ask"): the aim is to record smoking habits.

- Advise to give up smoking ("Advise"): information about possible tobacco-related diseases, the effects on diabetes, the development of possible benefits of abstaining from tobacco, and the recommendation to stop smoking. The recommendations should be adapted to the smoker's personal situation.

- Assessing the motivation to quit smoking ("Assess"): Ask about and quantify the motivation to stop smoking. Show the still-smoker the relevance of a possible change in behavior, name the risks of continuing smoking and show the possible benefits of a change.

- Offer support and referrals ("Assist"): Offer support for the patient's wish to stop smoking. Explain to the patient the advantages and disadvantages of the various therapeutic options for giving up tobacco and refer to help or support. Information should also be provided about possible withdrawal symptoms and their treatment.

- Arrange aftercare ("Arrange"): Follow-up appointments should be arranged at longer intervals for follow-up care. The aim is to provide support until abstinence is maintained.
Therapy for nicotine addiction

- Because of the increased health risks associated with tobacco addiction and its negative impact on the prognosis, especially for people with diabetes, the therapy for addiction is of particular importance here.

- The problem of smoking - especially with regard to the risk of diabetes-related or associated secondary and concomitant diseases - should be adequately addressed in the consultation. The consultation procedure described above can be helpful in practice for every diabetes patient who smokes (see info box "Consultation procedure").

- In addition to general explanations and information, psychoeducative measures, psychological/psychotherapeutic interventions and medication are used as therapeutic procedures.

- For people with diabetes, there are no long-term results on the effectiveness of drug-based nicotine replacement therapies for smoking cessation.

- Observe interaction: For the use of Champix ${ }^{\circledR}$ and Zyban ${ }^{\circledR}$ in persons with diabetes there are important restrictions on use or warnings which must be observed when prescribing.

\section{Schizophrenia}

According to the International Classification of Mental Disorders (ICD-10) of the World Health Organization (WHO), schizophrenia is classified in the $\mathrm{F} 2$ group

- The lifetime prevalence of schizophrenia in the general population is about $1 \%$.

- Manifestation of the disease is often preceded by a prodromal stage, in which initially unspecific, later more indicative symptoms are noticed.

- It is unclear whether the frequency of schizophrenia in people with diabetes mellitus is different from that of the general population.

- The prevalence rate of diabetes in patients with schizophrenia is about twice as high as in mentally healthy individuals. The prevalence of the metabolic syndrome is also increased.

\section{Interactions between schizophrenia and diabetes}

- The higher prevalence of diabetes mellitus and metabolic syndrome has a significant impact on the health of schizophrenic patients. Their life expectancy is significantly shortened.

- Cardiovascular diseases are a major cause of the shortened life expectancy, the development of which is considerably promoted by the higher prevalence of diabetes mellitus. The increased suicide rate of those affected contributes only to a lesser extent to the shortened life expectancy.

\section{Screening (diabetes)}

- Patients with schizophrenia and their relatives and caregivers should be informed about the risk of weight gain and diabetes mellitus.

- In patients with schizophrenia, examinations of body weight, waist circumference, blood pressure, fasting blood glucose and fasting blood lipids should be performed at the intervals specified in $\triangleright$ Tab. 2. 
- Tab. 2 Examinations to be performed regularly in patients with schizophrenia.

\begin{tabular}{|l|l|l|l|c|c|}
\hline Research & Start of therapy & After 4 and 8 weeks & After 3 months & Every 3 months & Annually \\
\hline Medical history & $\times$ & & & $\times$ \\
\hline Body weight (BMI) & $\times$ & $\times$ & $\times$ & $\times$ & $\times$ \\
\hline Waist circumference & $\times$ & & $\times$ & $\times$ \\
\hline Blood pressure & $\times$ & & $\times$ & $\times$ \\
\hline Fasting blood glucose & $\times$ & & $\times$ & $\times$ \\
\hline Fasting blood lipids & $\times$ & & \\
\hline
\end{tabular}

\section{Therapy}

\section{Therapy of schizophrenia}

- The therapy of schizophrenia follows a multimodal concept that includes pharmacotherapy, psychotherapy, sociotherapy, occupational therapy, patient training and therapeutical treatment with relatives.

- In patients with schizophrenia who already have diabetes mellitus or who are overweight/obese at manifestation, antipsychotics which are not associated with any weight gain should first be used. Weight gain is pronounced for the substances clozapine and olanzapine; intermediate weight gain can be expected for quetiapine and risperidone and low to no weight gain for amisulpiride, aripripazole and ziprasidone.

- If a pronounced weight gain or diabetes mellitus occurs with antipsychotic treatment, a dose reduction or change of the antipsychotic may be considered. The risks of such a measure, especially discontinuation of treatment with clozapine, with regard to possible exacerbation of schizophrenia, should be considered.

Therapy of obesity and diabetes

- In patients with schizophrenia, the prevention of weight gain should be given high priority in the treatment plan.

- Overweight patients with schizophrenia should be offered nutritional counseling and instructions for increased physical activity at the beginning of therapy and as it progresses.

- The treatment of diabetes mellitus should be integrated into the overall therapy concept of schizophrenia and current evidence-based diabetes guidelines should be followed.

\section{Depression}

The depressive symptoms are classified according to the International Classification of Mental Disorders (ICD-10) of the World Health Organization (WHO) as follows: depressive episode (F32.x), recurrent depressive disorder (F33.X), persistent affective disorders (F34.x), other or unspecified affective disorders (F38, F39), adjustment disorders (F43.2x). The depressive episode is the one most frequently associated with diabetes. In terms of differential diagnosis, depressive syndromes must be distinguished from depressive symptoms in schizophrenic disorders (F20), personality disorders (F6), post-traumatic stress disorders (F43.1) and schizoid-depressive disorders in schizoid-affective disorders.

- Depression is a risk factor for the development of type 2 diabetes. People who suffer from depressive symptoms have an increased incidence of type 2 diabetes.
- People with diabetes have an increased risk of developing depression by a factor of about 2 compared to the normal population.

- The prevalence of depression in type 1 and type 2 diabetes varies in controlled studies. It is higher in women compared with men, in clinical and in noncontrolled samples compared with population-based samples, and varies by disorder definition and instruments used.

- The risk of developing depression increases with the development and number of diabetes complications. Acute complications are associated with a higher rate of depression than chronic complications.

\section{Interaction between diabetes and depression}

Depression in people with diabetes is associated with:

- A significant reduction in the general and diabetes-specific quality of life and therapy satisfaction,

- A higher rate of functional limitations (including sick days from work),

- Lower adherence to therapeutic recommendations, especially nutritional recommendations,

- More frequent discontinuation of weight loss programs (overweight patients with type 2 diabetes),

- Greater frequency of nicotine addiction,

- Less sporting activity and movement,

- Less favorable metabolic control (HbA1c),

- A significantly-increased risk for the development of diabetes complications.

- A significantly increased mortality risk,

- Higher costs of medical care.

Depressive symptoms are also associated with numerous psychoneuroendocrinological changes, including activation of the innate immune system, alteration of the HPA axis, glucocorticoid receptor function, increased insulin resistance and activation of the autonomic nervous system.

\section{Screening}

- Only half of the depressions in people with diabetes are detected.

- People with diabetes mellitus should be screened for the presence of clinical or subclinical depression on a regular basis, at least once a year and during critical disease phases (diagnosis, hospitalization, development of sequelae, problematic disease behavior, impaired quality of life). 
- Patients suffering from a depressive disorder often consult their doctor because of unspecific physical complaints and trivialize the psychological symptoms. Weakness, increased fatigue, apathy, irritability, anxiety, sexual problems, sleep disorders, loss of appetite and weight loss can - in addition to the characteristic symptoms - be symptoms of depression.

- Depression should be considered for differential diagnosis in these nonspecific complaints.

- In cases of severe ketoacidosis or hypoglycemia, a differential diagnosis should be made to determine whether they are the expression of an attempt at suicide, for example in the context of depressive disorders or self-harming behavior.

- The central diagnostic instrument is the doctor-patient dialogue. During a patient-centered conversation, the physician should use screening questions for depressive disorders and ask about depressed moods, loss of interest and enjoyment in activities and the loss of motivation or drive in addition to questions on diabetes-specific stress. In practice, the two screening questions have proven to be effective (see info box “Depression screening").

- Depression screening questionnaire:

- WHO-Five Well-being Index (WHO-5) - integrated in the “Diabetes Health Passport” (Gesundheits-Pass Diabetes)

- Patient Health Questionnaire (PHQ-9)

- CES-D-Skala (Center for Epidemiological Studies Depression Scale)

- Beck Depression Inventory (BDI-II)

- Hospital Anxiety and Depression Scale (HADS-D)

\section{DEPRESSION SCREENING}

\section{Screening questions on depression}

Over the last 2 weeks, how often have you been bothered by the following problems?

1. Little interest or pleasure in doing things?

2. Feeling down, depressed or hopeless?

Evaluation: If one of the two questions is answered with "yes", depression is suspected and should be followed up with a detailed diagnosis

\section{Diagnostics}

- The following criteria are used to diagnose a depressive disorder and determine its severity according to ICD-10:

- Severity of the current episode

- Type and duration of depressive symptoms

- Occurrence of depressive episodes in the medical history

- Presence of a somatic syndrome

- Presence of psychotic symptoms (in severe episodes)

- The PHQ-9 questionnaire which includes the diagnosis criteria of depression can be used to support the physician-patient conversation.

- Since the depressive symptoms often develop in chronological context to other diabetes-related problems and complications, the correlation of the depressive symptoms with the diabetes-related somatic changes should be considered.
- Compared to the general population, the risk of suicide increases by a factor of 30 in depressed people. If there are signs of depression, the patient's suicidal tendency should be clinically assessed in every contact and, if necessary, asked about suicidal thoughts, impulses, and preparatory actions.

- Depression often occurs together with other mental disorders. The presence of two or more comorbid disorders in an individual usually complicates the course of the disease and makes therapy more difficult.

- Depression screening and structured depression diagnosis, while increasing the detection rate, only improve the treatment of depression when combined with structured treatment programs.

\section{Therapy}

- The following measures are available for the treatment of depression: Active watchfull waiting, psychotherapy, psychopharmacotherapy, combination treatment, light therapy, waking therapy, electroconvulsive therapy, sports and exercise therapy, occupational therapy or creative therapies supplement these procedures (see info box "Therapy steps for depression").

- Often, motivational work by the primary care physician or diabetologist is needed in advance of treatment for depression in people with diabetes. Therapy motivation is not a prerequisite for therapy but is often a (partial) success of a general practitioner or diabetological treatment. This is particularly effective if fixed cooperation structures between a medical or psychological psychotherapist, psychiatrist, primary care physician and diabetologist have been established.

- Psychotherapy should be offered to all patients with mild, moderate and severe depression, depending on the patient's preference. It can be offered to patients with adjustment disorders.

- The treatment of depression in people with diabetes should be delivered by therapists with psychological and diabetological knowledge, if possible.

- If there is diabetes related disstress, it should be taken into account in the psychotherapeutic treatment. Patients with diabetes mellitus, depending on their preference, should be offered antidepressant pharmacotherapy, especially in cases of moderate to severe depression.

- Antidepressants should not generally be used for the initial treatment of mild depressive episodes, but only after careful consideration of the benefit-risk ratio.

- In the presence of a mild depressive episode, antidepressant pharmacotherapy should only be suggested to the patient if the patient wishes to do so and if the patient has had positive experiences with treatment using antidepressants in the past, if the other treatment methods are not sufficient or if the patient suffers from recurrent depression with at least moderate episodes in the past.

- In cases of moderate and severe depression, the combination of pharmacotherapy with psychotherapy should be investigated.

- If pharmacotherapy is planned for a comorbidity of diabetes mellitus and depressive disorder, selective serotonin reuptake 
inhibitors (SSRI) should be offered. The side effects of tricyclic antidepressants, especially weight gain and blood glucose increase, should be weighed, monitored over time and only accepted if there is a specific indication for the use of tricyclics, such as in diabetic neuropathy. Changes in insulin sensitivity and blood glucose levels with antidepressant therapy are observed.

- Treatment should be carried out as part of an overall treatment plan that gradually integrates therapeutic options depending on the patient's motivation and preference, the severity of the depression and the conditions in which it develops.

- During the treatment of depression, regular monitoring of the treatment results should be carried out.

- If there are current problems with diabetes self-treatment (e. g. elevated HbA1 levels, hypoglycemia), diabetes training and treatment programs can be offered as complementary measures, especially for patients with mild and moderate depression, if they have not previously participated in a training program and are able to do so despite their depressive symptoms.

- Patients with diabetes and comorbid mild or moderate depression may be advised to use adapted physical training/ physical activity as an accompanying measure, taking into account possible contraindications.

\section{THERAPY STEPS FOR DEPRESSION}

\section{Mild depressive episode}

In the case of mild depressive disorders and adjustment disorders, the treating primary care physician, internist and diabetologist can initially carry out treatment as part of basic psychosomatic care. This includes:

- Establishment of a trusting, reliable and constant relationship with the patient, weekly appointment of the patient,

- Development of a common concept of illness, in which it is determined with the patient which factors lead to the development and maintenance of the depressive symptomatology and which are to be changed quickly,

- Information and education about depression and the relationship between depression and diabetes.

- Providing hope and encouragement,

- Relief from accusations, feelings of guilt and feelings of failure,

- Acceptance also of the complaining behavior of the patient and appreciation of his person,

- Active, flexible and supportive approach; anticipation of the patient's vulnerability,

- Dosed physical activity/movement instruction,

- Checking for suicidal tendencies.

- If there is no improvement of symptoms within 4 weeks, a psychotherapeutic treatment should be offered, for which the patient often has to be motivated first.

- If the focus is on processing diabetes-related stress, participation in a diabetes education and treatment program can be offered.

- Pharmacotherapy is not the primary focus for these severity levels of depression. However, if the patient has positive experience with antidepressant pharmacotherapy, if he or she suffers from a recurrent depressive disorder with at least moderately severe episodes in the past, or if the other therapeutic methods are not sufficient, antidepressant pharmacotherapy can also be offered.

- The course of the depression must be observed regularly.

\section{Moderate depressive episode}

- These patients require specific antidepressant treatment in addition to basic psychosomatic care. Both psychotherapy and drug-based antidepressant treatment should be offered to the patient and, depending on the patient's preference, should be carried out alternatively or in combination.

- Psychotherapy (behavioral therapy, psychotherapy based on psychoanalysis, interpersonal therapy) can be initiated with a medical or psychological psychotherapist.

- Dosed physical activity/movement instruction and recommandition for diabetes education if indicated.

- The response to drug therapy should be closely monitored so that the dosage can be adjusted as needed.

- If there is no marked improvement in symptoms after about four weeks (in the case of psychotherapy, the practitioner should adjust this time frame to the circumstances of the individual case), a referral to a specialist should be made.

\section{Severe depressive episode}

- In addition to the measures of basic psychosomatic care, a specialist should be integrated into the therapy of a patient with severe depression.

- The therapy preferably consists of a combination of pharmacotherapy and psychotherapy, whereby the preference and motivation of the patient is taken into account in the therapy decision.

\section{Continuous monitoring}

- In view of the chronicity of depressive disorders and the negative effects of even subclinical depression symptoms, the success of treatment should be continuously monitored in all phases of treatment.

- Achieving complete remission is the goal of depression treatment and should also be sought for patients with depression and diabetes.

\section{Anxiety Disorders and Diabetes-related Anxieties}

Most anxiety disorders in patients with diabetes are not specific to this patient group and can be classified according to the International Classification of Mental Disorders (ICD-10) of the World Health Organization (WHO). 
In addition, there are exclusively diabetes-related anxiety disorders, such as hypoglycemia anxiety, which cannot be assigned per se to a specific ICD-10 category, as they can meet the criteria for different mental disorders depending on how they surface.

- Hypoglycemia anxiety: excessive fear of possible future hypoglycemia. Typically, there are difficulties in differentiating between physical symptoms of anxiety and those of the adrenergic phase of hypoglycemia. To avoid possible hypoglycemia, significantly elevated blood glucose levels are usually accepted. Depending on the severity of the anxiety, the criteria for a "phobic disorder" (F40.0), a "panic disorder" (F41.0) or a "generalized anxiety disorder" (F40.1) may be met.

- Anxiety about the consequences of diabetes/anxiety about progression: disorder caused by excessive anxiety and worry about possible acute and long-term complications of diabetes. Depending on the severity of the anxiety, the criteria of a "generalized anxiety disorder" (F41.1), "anxiety and depressive disorder, mixed" (F41.2) or "adjustment disorder, anxiety and depressive reaction, mixed” (F43.22) may be met.

- About $20 \%$ of patients with diabetes show increased anxiety symptoms, without necessarily having an anxiety disorder in the sense of a clinical diagnosis. Anxiety disorders in patients with diabetes are about $20 \%$ more common than in the general population.

- The term "psychological insulin resistance" is used to describe the exaggerated fears of patients with type 2 diabetes of insulin treatment. In most cases, the criteria for a psychological disorder according to ICD-10 are not met. The main fears relate to injections and blood glucose monitoring, hypoglycemia, weight gain, complications, and concerns about stigmatization because of insulin injection treatment.

- Severe hypoglycemic events increase the probability of subsequent anxiety disorders in patients with type 2 diabetes. Single, unemployed women with diabetes complications have a higher risk for anxiety symptoms. In addition, anxiety correlates with higher age, lower weight and depression symptoms.

\section{Interaction between diabetes and comorbid anxiety disorders}

- Diabetes patients with an additional anxiety disorder are not only affected by their mental disorder. They also have an above-average burden both in dealing with their diabetes and in their general health-related quality of life.

- There is evidence that anxiety is associated with poor metabolic control, diabetes complications, mortality, mental comorbidity, poor quality of life and poor health status.

\section{Screening}

- The physician consultation is of central importance in the diagnosis of anxieties requiring treatment.

- Especially for patients who have intense or recurring concerns about their health and/or somatic symptoms, it is advisable to check whether some of these symptoms are caused by an anxiety disorder.

- Targeted screening questions are suitable for detecting anxiety disorders or pathological diabetes-related anxieties (see info box “Screening questions for anxiety disorders").

\section{SCREENING QUESTIONS FOR ANXIETY DISORDERS}

Based on the diagnostic short interview for mental disorders

- Panic disorder: Does it happen that you are suddenly and unexpectedly afraid without any real danger?

- Agoraphobia: Are you afraid or do you avoid certain situations and places such as department stores, driving, crowds, elevators or enclosed spaces?

- Social phobia: Are you afraid or do you avoid situations in which you could be observed or evaluated by other people, such as public speaking, gatherings, parties or conversations?

- Specific phobia: Are you afraid of or do you avoid situations such as injections, animals, heights, air travel, the sight of blood and injuries?

- Generalized anxiety disorder: Do you often suffer from unreasonably high levels of anxiety, for example about health-related family, professional or financial matters?

- Fear of diabetes-related complications and hypoglycemia are the most severe disease-specific stresses in people with diabetes. These can be a significant emotional barrier and can lead to problems in diabetes self-management. Syringe phobias only occur very rarely.

\section{SG QUESTIONS FOR DIABETES-RELATED PATHO- LOGICAL ANXIETIES}

- Hypoglycemia anxiety: Do you often suffer from severe worries about suffering a hypoglycemic event?... Quite independent of your normal target blood glucose: What is your personal "feel-good" blood glucose level? (Exaggeratedly high values, can give indications of hypoglycemia fears) ... Does it happen that you do not leave the house or avoid other situations for fear of hypoglycemia?

- Progression anxiety: Do you often suffer from unreasonable concern about the course of your diabetes?

\section{Questionnaire:}

- Patient Health Questionnaire (PHQ-D)

- Registration of fears of insulin therapy: Barriers to Insulin Treatment Questionnaire (BIT)

- Identification of pathological hypoglycemia anxiety: Hypoglycemia Fear Survey (HFS)

- Since a number of somatic disease factors can also cause anxiety symptoms (e. g. hyperthyroidism, migraine, coronary heart disease, asthma), a somatic differential diagnosis must be performed to exclude somatic causes. 
- In order not to promote hypochondriac anxiety, it is recommended, after an initial diagnostic clarification, to continue a more extensive somatic diagnosis only if there is a justified suspicion of certain organic diseases or resistance to therapy.

\section{Diagnostics}

Diagnosis of an anxiety disorder (

- Number, severity and duration of symptoms

- Extent of the individual burden caused by the disorder

- Impairment of the functional level

- Current diabetes-related and general developmental context of the anxiety disorder

- Previous anxiety disorders and possible treatment results

- If an anxiety disorder is diagnosed, screening for depression, somatoform disorders and substance abuse should also be carried out.

\section{Therapy}

- If a person with diabetes mellitus is diagnosed with an anxiety disorder or diabetes-related exaggerated anxiety, the diagnosis should be explained, and treatment offered ( $>$ Fig. 2). Every intervention requires continuous monitoring.

- Therapy recommendations for anxiety treatment based on findings in people without diabetes can (as long as there is no corresponding advance in the state of research) generally also be applied to people with diabetes (see info box "EXPLANATORY MODEL").

- Anxiety disorders or exaggerated diabetes-related fears that do not greatly impair the patient's functional level can be treated within the framework of primary care by psychoeducation and behaviorally-oriented bibliotherapy or Internetbased behavioral therapy for anxiety disorders.

- Anxiety disorders or diabetes-related exaggerated fears that have not responded to primary care measures, or moderate or severe anxiety disorder should be treated within the framework of outpatient psychotherapy. The first method of choice here is behavioral therapy. For generalized anxiety disorders, relaxation procedures can be recommended as an alternative or in combination.

- Anxiety disorders that have not responded to behavioral therapy should be treated by changing the therapy options. In addition to other psychotherapeutic methods (alone or in combination), psychopharmacological interventions are particularly suitable.

- If psychopharmacological therapy is offered, SSRI should be offered as the method of first choice. Benzodiazepines should only be offered for short-term crisis intervention. Neuroleptics should not be offered in the treatment of anxiety disorders.

- Complex, treatment-resistant severe anxiety disorders should generally be treated as inpatients or semi-inpatients as part of tertiary care. As a rule, psychopharmacological and psychotherapeutic measures should be offered in combination for this degree of severity.

- The therapy of anxiety disorders in diabetes should be carried out by therapists with psychodiabetological knowledge if possible.

\section{Medical anamnesis}

\section{Diabetes-related anxieties}

- Fear of the consequences of diabetes?

- Fear of hypoglycemia?

-Any fear/reservations about insulin? ...

\section{General anxiety}

- Panic attacks or chronic fear?

- Unexpected fears?

- Expectation anxiety and avoidance behavior?

- Impairments due to anxiety? ...

Questionnaires, if applicable

Anxiety disorders (independent of diabetes): PHQ-D

Diabetic-specific anxiety

Barriers of insulin therapy

Hypoglycemia anxiety

Exclusion of a medical disease factor

-Fig. 1 Algorithm for medical history/anxiety diagnosis in diabetes mellitus. 
Characteristics of anxiety disorder

\section{Level 4}

Complex treatment-resistant severe anxiety disorders which very severely impair the functional level

\section{Level 3}

Anxiety disorders that do not respond to level 2 interventions and severely impair the functional level or patient preference for drug therapy.

\section{Level 2}

Diagnosed anxiety disorders that do not remit after psychoeducation and active monitoring in primary care or moderate and severe anxiety disorders.

Level 1

All known or suspected minor anxiety disorders and/or diabetic-specific pathological anxiety (e.g. exaggerated hypoglycemia anxiety, fear of insulin, exaggerated fear of progression...).

\section{Therapy options}

Inpatient or semi-inpatient treatment in specialized hospitals. Usually more complex psychopharmacological and psychotherapeutic combination therapy, monitoring.

Recommendation for complementary psychopharmacological therapy. First method choice: SSRI. For unsuccessful behavioral therapy: in addition to medication, psychodynamic therapy, monitoring, if necessary.

Recommendation for outpatient psychotherapy with a medical or psychological psychotherapist (First choice: behavioral therapy).

For generalized anxiety disorder, additional relaxation techniques.

Monitoring of the further course.

Communicating the confirmed diagnosis and treatment options, developing a joint disease concept, providing hope and encouragement for dealing with mild phobias. If necessary, recommendation of behavior-therapeutically oriented self-help guidebooks, monitoring of further course.

- Fig. 2 Anxiety treatment stages for diabetes mellitus.

\section{EXPLANATORY MODEL}

Developing a common (simplified) explanatory model in primary medical care for co-morbidity of pathological anxieties in diabetes. The following questions can help to structure the discussion. If time is limited, the questions can be spread over several consultations:

1. "What has made the identified fears emerge or worsen?:" Biographical stress factors, patient attitudes that aggravate the problem, unfavorable previous experience with diabetes (such as severe hypoglycemia) can play a role here.

2. "What do you suppose has triggered or worsened the current fears?" It is often possible to identify current stress factors in the professional and/or private areas. Diabetes-related stress factors, such as the first occurrence or exacerbation of diabetes-related complications or the recommendation to initiate insulin treatment, can also trigger exaggerated fears in patients.

3. If the fears have been present for a longer time: "What conditions contribute to the maintenance of anxiety?"

If phobic fears are present, a more or less pronounced avoidance behavior will usually be observed in patients and which they exhibit severely as part of this anxiety disorder. In relation to diabetes, this can be, for example, the avoidance of normoglycemic blood glucose levels for fear of severe hypoglycemia. If the patient has a pronounced fear of diabetes worsening, the avoidance behavior can be expressed by neglecting important aspects of diabetes self-treatment in order not to have to deal with the frightening disease. Furthermore, catastrophic and irrational fears are often found especially in people with a panic or a generalized anxiety disorder; these fears can also be a chronic factor.

\section{Eating Disorders}

Eating disorders are classified according to the International Classification of Mental Disorders (ICD-10) of the World Health Organization (WHO) as follows: F50.0- * Anorexia nervosa (AN), F50.2- * Bulimia nervosa (BN). In the 4th edition of the American psychiatric classification scheme "Diagnostic and Statistical Manual of Mental Disorders" (DSM V) the "Eating Disorder Not Otherwise Specified" (EDNOS) is introduced.

In parallel, the ICD-10 (2) distinguishes "atypical bulimia nervosa" (F50.3); "eating attacks in other mental disorders" (F50.4) and "unspecified eating disorders" (F50.9).

- Currently, the prevalence for women with anorexia nervosa at risk age between 15 and 35 years is about $0.4 \%$. In the case of anorexia nervosa, there is often comorbidity with other mental illnesses, especially with depression, anxiety disorders or obsessive-compulsive disorders. 
- The prevalence of bulimia nervosa is higher in type 1 diabetes compared to anorexia nervosa and varies between 0.0 and $3.0 \%$.

- Data on the mean frequency of EDNOS in controlled studies vary between $3.0 \%$ and $9.0 \%$ in patients with type 1 diabetes.

- Data on the average frequency of insulin purging (intentional omission of insulin as a means of weight loss) varies between $5.9 \%$ and $39.0 \%$ depending on the study and region.

\section{Interaction between diabetes and eating disorders}

Eating disorders are associated with people with diabetes who:

- Are usually overweight and obese in type 2 diabetes,

- Have poor glycemic control in type 1 diabetes,

- Have a significantly increased risk for the development of diabetes complications (already in the case of pathological eating behavior without full-blown eating disorders; insulin purging),

- Have a significantly increased mortality risk in anorexia nervosa and continued insulin purging.

\section{Screening}

- In the general medical setting, an eating disorder should be considered in the following risk factors:

- Young women with low body weight

- Patients who present with weight worries, but are not overweight

- Women with cycle disorders or amenorrhea

- Patients who appear malnourished

- Patients with gastrointestinal symptoms

- Patients with repeated vomiting

- For the early detection of eating disorders in a general medical setting, the following two questions should be considered first:

- "Do you have an eating problem?"

- "Are you concerned about your weight or diet?"

- The Diab-Ess offers a specific validated screening tool for eating disorders in patients with diabetes.

- Various standardized questionnaires (e. g. Eating Disorder Inventory EDI, EDI-2, Eating Behavior Questionnaire [Fragebogen zum Essverhalten - FEV], Eating Disorder ExaminationQuestionnaire [EDE-Q]) allow for an eating disorder screening based on the information provided by the patient. It should be noted, however, that some of the items in these questionnaires refer to eating habits that are considered pathological in healthy individuals but are considered adequate in people with diabetes (e. g., constant monitoring of food intake). This ultimately carries the risk of false positive results.

\section{Diagnostics}

- The comorbidity of diabetes and anorexia nervosa is extremely rare and usually easy to diagnose due to the cachectic nutritional status of the anorexic patient.
- In every patient with inadequate metabolic control and with significant fluctuations in blood glucose levels and weight - especially in girls in adolescence and young women - a bulimia nervosa with or without insulin purging should be considered and differentially diagnosed at an early stage.

- For the diagnosis of an eating disorder, a detailed biographical history is often necessary. Patients have the tendency to deny eating disorders for years and this anamnesis can help the patient overcome personal reservations and summon the courage to face the disorders.

\section{Therapy}

- Psychotherapeutic interventions to treat eating disorders are effective. There are no contraindications for use in patients with diabetes. The treatment of eating disorder patients with type 1 diabetes should therefore be carried out according to the current S3 guideline "Diagnosis and treatment of eating disorders".

- The first choice of therapy is psychotherapy.

- The treatment can be disorder-oriented and take into account the physical aspects of the disease.

- Professional psychotherapeutic treatment of these patients is recommended because of health risks of the eating disorder and the increased risk of mortality, especially of anorexia nervosa, the frequently encountered comorbid depressive disorder and the negative impact on diabetes therapy.

- The therapy of eating disorders in people with diabetes should be carried out by therapists with psychodiabetological knowledge if possible.

- Outpatient, semi-stationary and inpatient treatment can take place in institutions or with medical or psychological psychotherapists who have expertise in the treatment of eating disorders and who provide disorder-oriented therapy elements.

- It should be taken into account that the healing process usually takes many months of treatment.

- In bulimia nervosa, SSRIs are the drug therapy of choice.

- Only fluoxetine is approved in Germany in combination with psychotherapy for adults with bulimia nervosa.

- Psychoeducative approaches alone are not sufficient or appropriate.

- Successful psychotherapy requires an understanding of the life situation of the patient in general and especially of the patient with diabetes. This requires particular knowledge on the part of the treating psychotherapist regarding the therapy regime and its possible connections with eating behavior/ eating disorders (e. g. hypoglycemia, physical activity, etc.).

- Type 2 diabetes patients with eating disorders usually suffer from binge eating and obesity, so considerations about all three disease entities should be included in the treatment. A multimodal treatment concept with psychotherapy and weight management as integral components is therefore appropriate.

- Closed cooperation between the diabetological and medical/ psychological-psychotherapeutic treatment team is necessary. 


\section{Psychological or behavioral factors in diabetes}

Chronic stress, interpersonal problems, lack of social support and problems in coping with the disease can have significant relevance for the quality of life and metabolic regulation in people with diabetes.

If these or other factors are important for the beginning, but especially for the development of diabetes, they can be classified according to the corresponding history under diagnosis F54 (ICD 10) ("Mental factors or behavioral influences in diseases classified elsewhere").

Thus, these factors can also provide cause and justification for a psychological intervention regardless of the presence of a mental disorder.

\section{Interaction between chronic stress, interpersonal problems interpersonal or coping problems and diabetes and diabetes}

- Chronic stress (e. g. emotional stress in work and private life) can promote the manifestation of type 2 diabetes.

- In manifested diabetes, metabolic control and quality of life can also be negatively affected.

- Lack of social support or interpersonal problems can also be a barrier to a good quality of life and proper metabolic control. Here, a distinction must be made between an actual lack of social support (e.g. after the loss of a partner) and dysfunctional support where, for example, overprotectiveness hinders the patient from taking on necessary personal responsibility.

- In addition, interpersonal problems such as chronic conflicts can largely block any potential for social support within the framework of a relationship. A more suitable measure for estimating actual social support than the mere availability of persons is how the patient (subjectively) perceives support received by third parties.

- Problems with coping with the demands of the disease often occur during the course of the disease and can negatively influence the therapeutic behavior and glycemic control.
- Patients with diabetes complications are at particularly high risk of experiencing problems in coping with the disease.

\section{Screening}

- If there is evidence of diabetes-related stress, a validated questionnaire (e.g. PAID) can be used.

\section{Diagnostics}

Psychological or behavioral problems in diabetes do not justify assignment to another category in Chapter V (ICD-10). An additional code should be used to identify the physical disorder (e. g. diabetes).

\section{Interventions}

- Positive effects of various psychological interventions for F 54 disorders on glycemic control and quality of life have been documented. The effects were generally detectable independently of any diabetes education that may have been conducted at the same time.

- Interventions to reduce stress should be offered to patients with severe stress and inadequate glycemic control and/or reduced quality of life.

- Targeted psychotherapeutic interventions may be considered for patients with serious interpersonal problems and/or low social support that have a negative impact on diabetes therapy.

- Psychological interventions to promote disease management should also be offered - independent of training - to patients with serious problems managing the disease.

- If there are psychological or behavioral influences that have a serious effect on the disease, therapy F54 should be carried out by therapists with psychodiabetological knowledge if possible.

\section{Conflict of Interest}

The authors state that there is no conflict of interest. 